\title{
Prioritizing Rare Diseases: Psychological Effects Influencing Medical Decision Making
}

\author{
Johanna Wiss, Lars-Åke Levin, Andersson David and Gustav Tinghög \\ Journal Article
}

Tweet

N.B.: When citing this work, cite the original article.

Original Publication:

Johanna Wiss, Lars-Åke Levin, Andersson David and Gustav Tinghög, Prioritizing Rare Diseases: Psychological Effects Influencing Medical Decision Making, Medical decision making, 2017.

http://dx.doi.org/10.1177/0272989X17691744

Copyright: SAGE Publications (UK and US)

http://www.uk.sagepub.com/home.nav

Postprint available at: Linköping University Electronic Press

http://urn.kb.se/resolve?urn=urn:nbn:se:liu:diva-134647

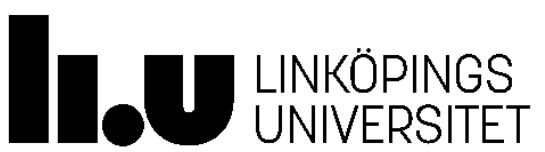




\section{Prioritizing rare diseases: Psychological effects influencing medical decision making ${ }^{1}$}

Wiss Johanna ${ }^{1}$, MS, Levin Lars-Åke ${ }^{2}, \mathrm{PhD}$, Andersson David ${ }^{3}, \mathrm{PhD}$, \& Tinghög Gustav ${ }^{1,3}, \mathrm{PhD}$

1. The National Center for Priority Setting in Health Care, Department of Medical and Health Sciences, Linköping University, SE-581 83 Linköping, Sweden.

2. Center for Medical Technology Assessment, Department of Medical and Health Sciences, Linköping University, SE-581 83 Linköping, Sweden.

3. Division of Economics, Department for Management and Engineering, Linköping University, SE-581 83 Linköping, Sweden.

\footnotetext{
${ }^{1}$ Financial support for this study was provided in part by a grant from The Swedish Association of the Pharmaceutical Industry (LIF) and the Ragnar Söderberg Foundation. The funding agreement ensured the authors' independence in designing the study, interpreting the data, writing, and publishing the report.
} 


\section{Abstract}

Background: Measuring societal preferences for rarity has been proposed to determine whether paying premium prices for orphan drugs is acceptable. Objective: To investigate societal preferences for rarity and how psychological factors affect such preferences. Method: A postal survey containing resource allocation dilemmas involving patients with a rare disease and patients with a common disease, equal in severity, was sent out to a randomly selected sample of the population in Sweden (return rate $42.3 \%, \mathrm{n}=1270$ ). Results: Overall, we find no evidence of a general preference for prioritizing treatment of rare disease patients over common diseases patients. When treatment costs were equal most respondents (42.7 \%) were indifferent between the choice options. Preferences for prioritizing common disease patients over rare disease patients were more frequently displayed (33.3 \% vs. $23.9 \%$ ). This tendency was, as expected, amplified when the rare disease was costlier to treat. The share of respondents choosing to treat the rare disease patients increased when presenting the patients in need of treatment in relative rather than absolute terms (proportion dominance). Surprisingly, identifiability did not increase preferences for rarity. Instead, identifying the rare disease patient made respondents more willing to prioritize the common disease patients. Respondents' level of education was significantly associated with choice - the lower the level of education the more likely they were to choose the rare option. Conclusion: We find no support for the existence of a general preference for rarity when setting health care priorities. Psychological effects, especially proportion dominance, are likely to play an important role for when preferences for rarity are expressed.

Key words: Orphan drugs, rare diseases, priority setting, societal preferences, survey, resource allocation, proportion dominance, identifiability 


\section{Introduction}

Should the rarity of a condition be taken into account when allocating resources in health care? Regulations to stimulate research and development of rare disease treatments have largely increased the number of orphan drugs [1, 2]. Meanwhile, orphan drugs have commonly shown a higher cost per health gain compared to other drugs [3]. Despite not qualifying as cost-effective, many orphan drugs are subsidized in the EU member states. Consequently, priority setting regarding orphan drugs has developed into a key issue in the discussion on whether publicly funded health care systems should be willing to pay more per health gain for some patients. It has been argued that it is unethical to value health gains more highly for patients suffering from rare diseases compared to patients with common diseases. Providing non-cost-effective orphan drugs would lead to reduced access to cost-effective treatments for other patients suffering from equally severe common diseases $[4,5]$. The key policy (and ethical) question is: should society value the health gain differently depending on whether a patient suffers from a rare or a common disease? A way of informing policy discussions and potentially justifying exemptions for rare diseases from standard practice when implementing new treatments is to examine if there are societal preferences for doing so.

In this study we build on the design introduced in the seminal work by Desser et al. [6-8], who, in a Norwegian sample, found no strong support that there exists a societal preference for rarity. This was shown both in scenarios where treatment costs were described as equal for the rare and the common disease and in scenarios where the rare disease was costlier to treat (reflecting real world opportunity costs). Respondents, however, expressed a strong general support for statements concerning equal right to treatment for patients with rare diseases. This study extends past research on general societal preferences for rarity by also exploring if such preferences are malleable to a set of psychological factors [9-12].

\section{Psychological effects in medical decision-making}

Based on literature on psychological factors influencing helping behavior, we set out to explore four aspects related to how information is presented that are likely to influence preferences for rarity: (1) if the decision is made with respect to a group of patients or an individual patient ("level of decision-making"), (2) if the patient is identified with name and a picture ("identifiability") (3) if the number of patients treated is expressed in absolute or in relative terms ("proportion dominance"), and (4) if the scenario is expressed as giving or denying treatment to the patients ("attribute framing"). We hypothesize that each of these aspects will have an impact on preferences for rarity, demonstrating the context-dependency of preferences. Further, we explore whether the impact of these aspects are similar when treatment costs are higher for the rare disease. 


\section{Level of decision-making}

Priority setting takes place on many different levels of the health care organization - from decisions made by the physician directly in relation to a patient (bedside rationing) to high level policy decisions (desktop rationing) [13]. Redelmeier and Tversky [9] showed that physicians, as well as lay people, make different decisions when evaluating an individual patient than when considering a group of comparable patients. More specifically, physicians gave more weight to efficiency concerns at the group level. Previous studies have also shown that an individual, in contrast to a group, is viewed as a psychologically coherent unit and that this leads to stronger impressions about individuals than groups [14]. Policy decisions regarding rare disease patients, often in need of specialized care, are more likely to concern individual patients rather than groups. We therefore seek to examine whether the perceived differences between individuals and groups leads to people prioritizing rare and common diseases differently. We predict that preferences for rarity will be more pronounced when making priority setting decisions at the individual level.

\section{Identifiability}

Closely linked to the level of decision-making is the identifiability of a patient. People tend to be more willing to help individuals that are presented as identified rather than anonymous (i.e. the identifiable victim effect). This effect has been shown in numerous studies on helping behavior [15-19]. Moreover, people often feel a sense of a moral duty to help identified individuals presenting themselves to health services with a severe, life-threatening condition. The feeling of moral obligation towards a single identified individual, regardless of cost, is often termed the rule of rescue [10, 20]. Media coverage and the public debate on reimbursement decisions regarding orphan drugs commonly involve a reference to the rule of rescue. Mackenzie et al. [21] analyzed the media coverage preceding the funding decision regarding the drug Herceptin (Trastuzumab), for women suffering from HER2 breast cancer in Australia. They found that a majority (54\%) of the news statements featured "desperate, sick women in double jeopardy because of callous government/incompetent bureaucracy”. The straightforward prediction based on previous literature on the identifiable victim effect and rule of rescue is that preferences for rarity should increase when presenting a patient with a name and a picture. However, it should also be noted that the tendency to offer greater aid to specific identified victims has been shown to be context dependent [22].

\section{Proportion dominance}

A preference for maximizing relative savings at the expense of absolute savings is commonly referred to as "proportion dominance" [11, 23-25]. Individuals generally prefer to help a larger proportion (e.g. 100 out of 100 people) rather than a smaller proportion (e.g. 100 out of 10000 people) even though the number of people helped is identical. The proportion dominance effect is often linked to a drop in the bucket-feeling implying that people are tempted to shut down 
emotionally and ask “what is the point?” when facing problems of large magnitude [25]. This indicates a tendency among people to prefer actions that completely eliminate a problem over actions that only eliminate some part of a problem. Accordingly, people have a general preference for dealing with smaller problems before moving on to bigger problems, thereby sometimes ignoring efficiency concerns.

The effect of proportion dominance is likely to be important when setting priorities for patients with rare diseases given that the relative share of patients with a rare disease that can be treated is bound to be higher than the relative share of patients with a common disease that can be treated. For example, if we assume that costs and effects for treating rare and common disease patients are identical, we can treat 80 out of 80 rare disease patients but only 80 out of 10000 common disease patients. Being able to treat a higher proportion of patients with rare diseases could potentially create preferences for rarity in order to avoid a drop in the bucket-feeling.

Importantly, to avoid this feeling, people might also be willing to sacrifice overall efficiency [24], e.g. prioritize the health gain for 80 out of 80 patients rather than for 100 out of 10000 patients. These tendencies among people are why we predict that expressing health gains in relative rather than absolute terms will increase the share of respondents choosing to treat the rare patient group even when this implies sacrificing overall efficiency.

\section{Attribute framing (giving vs. denying treatment)}

Framing equivalent decision problems as either gains or losses has been shown to affect choices. Tversky and Kahneman [12] described the Asian disease problem - a scenario where participants could choose between two medical programs to combat an unusual disease. One program was described as a secure option (some lives will be saved for sure) and one program as a risky option (some probability that everyone will be saved and some probability that no one will be saved). Two versions of the scenario were randomly allocated to participants: one version where the outcomes were expressed in lives saved and one version where the outcomes were expressed in expected deaths. Results showed that presenting outcomes either in terms of gains or in terms of losses led to a reversal of preferences for the otherwise identical medical programs. In this study we employ a case of attribute framing where the decision problem is presented in positive or negative terms, i.e. either giving or denying treatment.

When discussing resource allocation in health care on a policy level, a give-frame is typically referred to as priority setting while a deny-frame is typically referred to as rationing. It is probable that rationing evokes negative emotions in individuals as it associates with a situation of scarcity and forced choice. In contrast, priority setting is likely to be regarded in a more positive way, a situation where informed choices are made to benefit the society at large. In the public debate regarding orphan drugs, focus is often on cases where patients have been denied a treatment. Given that denying (rationing) care is likely to be a more emotionally burdensome decision it is not farfetched to believe that this framing could increase preferences for rarity. 


\section{Method}

\section{Survey methods}

A postal questionnaire was sent out to a randomly selected sample of the population in Sweden (living in the county of Östergötland) aged between 20 and 75 years. A total number of 3000 questionnaires were distributed. Two reminders were sent out. Before sending out the survey it was pilot tested and respondents were interviewed to make sure instructions and scenarios were interpreted as intended.

\section{Survey design}

To introduce the topic to respondents, the questionnaire started with a short description of rare diseases and why treatments aimed at patients with rare diseases might be challenging when setting priorities in health care. This information was kept as short and neutral as possible to avoid influencing respondents' choices in the following scenarios.

Figure 1 gives a general overview of the questionnaire, where variations in choice options are presented for each scenario. The questionnaire was designed to allow for both within- and between-subjects comparisons. Thus, three different versions of the questionnaire were sent out (version A-C).

\section{Within-subject comparisons}

Each questionnaire contained four allocation scenarios where participants were asked to make a choice between allocating health care resources to patient(s) with a rare disease, patient(s) with a common disease or to be indifferent. The rare and the common diseases were described as equal in severity using EQ-5D health state descriptions.

In scenario 1 and 2, respondents were asked to allocate health care resources to either a group of 100 patients with a rare disease or a group of 100 patients with a common disease. In scenario 3 and 4 , respondents were asked to allocate health care resources to either one patient with a rare disease or one patient with a common disease. We label scenario 1 and 2 group-level scenarios and scenario 3 and 4 individual-level scenarios.

The costs for treating the rare and the common disease patient(s) were described as equal in scenario 1 and 3, whereas the costs for treating the rare disease patient(s) were increased in scenario 2 and 4 . We label scenario 1 and 3 equal-cost scenarios and scenario 2 and 4 unequalcost scenarios. The increased opportunity cost for treating the rare disease patients reflect the higher costs of rare disease treatments compared to common disease treatments.

The exact phrasing of scenario 1 (group level, equal cost) was as follows: 
Imagine that the county council has obtained additional resources that can be used to treat 100 patients. The county council must decide which one of two similar diseases that should be treated: Disease A, a rare disease (100 cases per year in Sweden), or Disease B, a common disease (10 000 cases per year in Sweden). Disease A and B are equally severe. Patients can walk with some difficulty and they suffer from severe pain. Both patient groups will regain full health with treatment. The costs of treating patients with Disease A and Disease B are identical. How do you think that the additional resources should be used?

The respondents were then asked to choose one of the following options: to treat 100 patients with Disease A (rare disease), to treat 100 patients with Disease B (common disease) or to be indifferent.

Scenario 2 (group level, unequal cost) was also described as a group-level decision, but where the cost of treating a patient with a rare disease was eight times higher than treating a patient with a common disease. Respondents were asked to make a choice between treating 100 patients with a rare disease, 800 patients with a common disease, or to be indifferent.

Scenario 3 (individual level, equal cost) was presented as an individual level decision where respondents were asked to make a choice between treating Patient $\mathrm{X}$ with a rare disease or to treat Patient $\mathrm{Y}$ suffering from a common disease or to be indifferent.

Scenario 4 (individual level, unequal cost) was presented as an individual level decision, but with the cost of treating Patient $\mathrm{X}$ (rare disease) being eight times higher than treating Patient $\mathrm{Y}$ (common disease). Respondents were asked to make a choice between treating Patient $\mathrm{X}$ (rare disease), Patient $\mathrm{Y}$ (common disease) plus seven other patients also suffering from the common disease, or to be indifferent.

For full description of all scenarios see appendix.

\section{Between-subject comparisons}

To test for differences in respondents' preferences for rarity due to information presentation, three versions (A-C) of the questionnaire were randomly distributed. Scenarios 1-4 were identical across versions A-C, except for minor variations in the way information was presented. The variations in information, related to the effects described in the introduction, were the following:

i) Identifiability: The patient suffering from the rare disease was presented either with a picture and a name or as an anonymous patient without picture and name.

ii) Proportion dominance: The number of patients possible to treat was expressed either in absolute terms (i.e. treating 100 rare patients vs. treating 100 common patients) or as a proportion (i.e. treating 80 out of 80 rare patients vs. treating 100 out of 10000 common patients). Thus, the scenario used to test for the proportion dominance effect 
(i.e. scenario 1 in survey version $\mathrm{C}$ ) did not reflect equal cost between rare and common disease.

iii) Attribute framing: The respondents were asked to either give priority to one patient or deny and ration care to one patient.

\section{Results}

Data was collected between April and June 2014. Return rate was $42.3 \%$ ( $n=1270)$. Respondent characteristics regarding age, gender, education and monthly salary were fairly representative compared to the Swedish population. Mean age among respondents was 50.9 years and $53.9 \%$ of subjects in the sample were women. There were no significant differences between survey versions regarding age, gender, education or monthly income. See appendix for full description.

\section{General preferences for rarity}

Figure 2 shows the proportion of respondents that chose to allocate funds to rare or common disease patient(s) for the four scenarios across all versions (A-C) of the survey. It also shows the fraction of respondents who stated that they were indifferent between the two choice options. The two left clusters of bars in figure 2 show responses when treatment costs were described as equal. At the group-level, where respondents faced a decision to fund treatment for either 100 patients with a rare disease or 100 patients with a common disease, $23.9 \%$ of the subjects chose to treat the rare disease group. A larger share, $33.3 \%$, chose the common disease group while $42.7 \%$ were indifferent. Thus, a larger proportion of the respondents prioritized the common disease group when treatment costs were described as identical. At the individual-level, where respondents faced a decision to fund treatment for either one patient with a rare diseases or one patient with a common disease, $23.0 \%$ of the subjects chose to treat the rare disease group. A smaller share, $18.5 \%$, chose the common disease group while $58.5 \%$ were indifferent. Thus, there were notable differences in responses between the individual-level compared to the grouplevel. A logistic panel regression revealed that age and gender had no effect on preferences for rarity whereas level of education had a significant effect. The odds for prioritizing rare disease patient(s) were almost double for respondents who had only finished primary education compared to respondents who had completed three years of higher education $(\mathrm{OR}=1.82, \mathrm{p}<.01$, see appendix for full analysis).

The two right clusters of bars in figure 2 show responses in the scenarios where treatment costs between rare and common diseases were unequal. More specifically they show responses in the scenarios where it was eight times costlier to treat the rare disease patient(s) than the common disease patient(s). As expected, a majority of respondents, $64.8 \%$, chose to maximize the number of patients treated and prioritized the common disease while only $13.3 \%$ chose the rare disease patients at the group-level. The results were similar at the individual-level, where $67.2 \%$ chose to 
treat the common disease patients and $10.9 \%$ the rare disease patient. Similar to the equal-cost scenarios, a logistic panel regression revealed that age and gender had no effect on preferences for rarity, whereas level of education had a significant effect. This effect was even more pronounced in the unequal cost scenarios compared to the equal cost scenarios. Respondents with only primary education had almost four times higher odds for prioritizing rare disease patient(s) compared to respondents with higher education $(\mathrm{OR}=3.70, \mathrm{p}<.01)$.

As could be expected, the fraction of respondents choosing to prioritize the rare disease patient(s) decreased in the unequal cost scenarios compared to the equal cost scenarios, both at the individual (23.0 \% vs. $10.9 \%$ ) and at the group level (23.9 \% vs. $13.3 \%$ ) (McNemar's test, $\mathrm{p}<.01)$. Consequently, the scenarios that are arguably closest to the real world decision problem in orphan drug funding were also the ones where support for prioritizing rarity was the lowest.

\section{The effect of the level of decision making, identifiability, proportion dominance and attribute framing on preferences for rarity}

Figures 3a-d show how preferences for treating the rare disease patient(s) changed with regards to key psychological manipulations. Logistic regression analyses confirmed the results below, see appendix.

The percentage of respondents that chose to treat the rare disease patient(s) at different levels of decision making is shown in figure 3a. As opposed to the results shown in figure 2, the results depicted in figure 3a represent within-subject differences only from the baseline version of the questionnaire. When costs were equal, $19.2 \%$ allocated funds to the rare patient at the group level. This proportion of subjects increased to $23.6 \%$ at the individual level (McNemar's test, $\mathrm{p}=.09$ ). In the unequal cost scenarios, the effect of moving from group to individual level of decision making was in the opposite direction. The proportion of respondents giving priority to rarity decreased from $11.9 \%$ to $8.9 \%$. This decrease in preferences for rarity was statistically significant (McNemar's test, $\mathrm{p}<.05$ ).

The percentage of respondents that chose to treat the rare disease patient dependent on identifiability is depicted in figure 3b. Identifying the rare disease patient did not increase the share of respondents choosing to allocate resources to the patient in either the equal (Chi-Sq, $\mathrm{p}=.77$ ) or the unequal cost scenario (Chi-Sq, $\mathrm{p}=.89$ ). Although the identifiability had no effect on

preferences for rarity per se, in the unequal cost scenario fewer subjects were indifferent and more prioritized the common disease patient when the rare disease patient was identified rather than not identified (Chi-Sq, $\mathrm{p}<.05$ ). Thus, there was an indication that identifiability had a negative effect on preferences for rarity when costs were unequal.

The effect of the proportion dominance manipulation is shown in figure 3c. In the equal cost scenario, the share of respondents who stated a preference for rarity increased from $19.2 \%$ to $28.2 \%$ when outcomes were presented in relative terms (i.e. treating 80 out of 80 rare patients or 
100 out of 10000 common patients), compared to when outcomes were presented in absolute terms (i.e. treating 100 rare or 100 common patients). This effect was statistically significant (Chi-Sq, $\mathrm{p}<.01$ ). Thus, more participants chose to maximize the relative number of treated patients, so called proportion dominance, at the expense of the absolute number of treated patients. In the unequal cost scenarios, stated preferences for rarity increased from $11.9 \%$ to 14.7 $\%$ (Chi-Sq, $\mathrm{p}=.24$ ). Thus, the effect of proportion dominance becomes less prevalent as opportunity cost increases. Still, it should be noted that rational choice theory [26] predicts that the share of participants that allocate funds to the rare disease group should be lower (not higher!) in the scenarios testing for proportion dominance, since allocating funds to treating common patients maximizes the number of treated patients.

The percentage of respondents that chose to treat the rare disease patient dependent on attribute framing is depicted in figure $3 \mathrm{~d}$. In the equal cost scenario, when asking respondents to deny funding to patients as opposed to prioritize who should receive funding, the percentage choosing the rare disease patient decreased from $23.6 \%$ to $20.7 \%$ (Chi-Sq, p=.34). In the unequal cost scenario, the share of respondents who had a preference for rarity increased from $8.9 \%$ when prioritizing to $15.1 \%$ when rationing care. This increase was statistically significant (Chi-Sq, $\mathrm{p}<.01)$.

\section{Discussion}

Setting priorities in health care presents decision makers with ethical and economic challenges. One particularly difficult question facing health care decision makers is how to balance concerns for equal access to care with concerns for health maximization. The prominent health policy debate about whether society should give special considerations for drugs to treat rare diseases highlights this conflict in practice. Overall, this study finds that people do not want to prioritize treatments for patients with rare diseases over common diseases. Most respondents (42.7 \%) were indifferent when asked to prioritize between rare and common disease patients when treatment costs were described as equal. This is not surprising since respondents who adhere to costeffectiveness as a guiding principle should state indifference when costs and benefits are identical. Moreover, many people typically refuse to engage in trade-off thinking when it comes to health and wellbeing [27]. Among respondents who stated a preference for either the rare or the common disease patients, preferences for treating the common disease were greater (common disease: $33.3 \%$, rare disease: $23.9 \%$ ). This result could be interpreted as a general expression of self-interest, since individuals prioritize the disease they are more likely to experience. The tendency to prioritize the common disease patients was, as expected, amplified when treatment costs were described as higher for rare disease patients than for common disease patients. Our general findings are in line with previous studies from Norway and Canada $[6-8,28]$ that also 
found that preferences for common disease patients over rare disease patients were more commonly displayed than vice versa. Moreover, discrete choice experiments in the UK found no evidence in support for claims that governments should grant special funding status for orphan drugs [29, 30]. Thus, there is now converging evidence from different countries that rarity alone is not an attribute people in general think should influence reimbursement decisions in health care.

A second aim of this study was to investigate to what extent preferences for rarity are malleable to a set of psychological factors. Although we find no indication of a general preference for rarity, our results show that psychological factors, especially proportion dominance, cause preferences for rarity to fluctuate. Although the proportion dominance effect has received considerable attention in other research areas related to judgment and decision making, it has previously been largely neglected in the context of health care priority setting. In line with our prediction, the share of respondents choosing to treat the rare patient group increased when the choice options explicitly stated that the entire rare disease group could be treated (80 out of 80 patients) versus only a fraction of the common disease group (100 out of 10000 patients), even though this implied a loss in overall efficiency. There are several potential explanations for why proportion dominance causes preferences for rarity to increase. Preventing the deaths of 80 out of 80 patients (i.e. saving the entire group) may seem more urgent than 100 out of 10000 patients (i.e. saving only a fraction of the group). Although a greater absolute number of patients are treated in the latter case, respondents perceive the option as less attractive when only a fraction of the group can be saved. A preference for equality is another reason for why people may be influenced by proportion dominance. Treating 80 out of 80 patients implies treating all patients in the group, whereas treating 100 out of 10000 patients' is a choice where a large share of patients will not access treatment. In health policy, there are at least two ways in which proportion dominance can influence decisions regarding funding of orphan drugs. First, policy makers may want to eliminate the "smaller" problems before they move on to the "bigger" ones. Second, compared to the total health care budget, the cost of treating a rare disease is relatively small. Paying for rare disease treatments, even though very expensive and non-cost-effective, may be perceived as having a limited impact in relation to the total health care budget. This would result in a case of cumulative cost neglect, where policy makers tend to overlook the aggregate outcome of many inefficient decisions.

The prediction that identifiability should increase preferences for rarity was not supported. Instead, identifiability had no effect on preferences for rarity. However, identifying the rare disease patient when costs were unequal increased the share of respondents prioritizing the common disease, indicating a negative effect of identifiability on preferences for rarity. Although we did not predict this negative effect of identifiability on preferences for rarity, it is consistent with a recent study showing that the effect of identifiability is sometimes negative in a Swedish context [31]. It is possible that the effect of identifiability would have been positive had we used a separate evaluation design where individuals were not explicitly aware of the alternative use of 
resources. In cases where trade-offs are not explicit, identifiability is more likely to have a positive effect on allocation decisions. Thus, a difference between identifiability in this study and the identified patients brought forward by patient advocates, pharmaceutical companies and the media is the presentation (or lack thereof) of alternative costs [32]. It should also be mentioned that the choice of person depicted in the picture could also play a role. The picture used in this study was of a middle aged, black male with a neutral facial expression.

The results related to "level of decision making" and "attribute framing" are hard to interpret, especially since the effects were in opposite directions depending on whether the costs were described as equal or unequal. For attribute framing the effect went in the predicted direction in the unequal cost scenario. Preferences for rarity increased when decisions were framed as rationing care rather than giving priority. For level of decision making the effect went in the opposite direction of what would be expected in the unequal cost scenario. Preferences for rarity were higher for group-level decisions compared to individual-level decisions. However, given that we had no strong a priori hypotheses in these cases it is possible that the findings related to level of decision making and attribute framing are false positives. The results should therefore be interpreted with caution unless confirmed in future studies. Our use of a joint evaluation design, where options are evaluated simultaneously, instead of a separate evaluation design, where each option is evaluated in isolation, could be a contributing factor to our puzzling results. In real life most evaluations are made in isolation. When evaluating both options at the same time respondents are likely to reflect more, thereby limiting the influence of emotions on decision making. Redelmeier and Tversky [9] used a separate evaluation design when showing that physicians were more likely to give extra attention to an individual patient than a group of patients. In this study we wanted to be explicit about the opportunity cost of treating the rare disease patient in order to explore more informed decision making.

Finally, some general limitations of this study should be acknowledged. First, we had a fixed order of presentation for the scenarios in the survey and could therefore not control for potential effects of order. Second, and similarly to other studies exploring stated preferences, the hypothetical nature of the survey may reduce the reliability of the responses. Third, and perhaps most importantly, there are additional factors, unexamined in our study, that could influence preferences for reimbursing orphan drugs. These include differences in disease severity, whether the treatment addresses an unmet need and whether people value research and development for orphan drugs more highly than for other drugs. For future research it would be beneficial to complement our experimental approach with a qualitative approach in order to get a more nuanced understanding of how preferences related to rare diseases and orphan drug reimbursement are manifested.

To conclude, we find no evidence that there exists a general preference for rarity among the general population when setting health care priorities. Psychological factors, however, cause preferences for rarity to fluctuate but the share of people who state preference for rarity is always 
below $50 \%$.

\section{Acknowledgements}

We thank Daniel Västfjäll and Marika Wenemark for assistance in the design of the questionnaire, Lars Valter for helping with the sample selection, and Lina Koppel, Arvid Erlandsson, and Paul Slovic for giving valuable feedback on earlier versions of the manuscript. We also thank three anonymous reviewers for giving insightful comments and suggestions that substantially improved the manuscript. Financial support by The Swedish Association of the Pharmaceutical Industry (LIF) and the Ragnar Söderberg Foundation is gratefully acknowledged. 


\section{References}

[1] US Food and Drug Administration. Orphan Drug Act. 1983.

[2] European Commission. Regulation No 141/2000 of the European Parliament and of the Council of 16 December 1999 on orphan medicinal products. In: Official Journal of the European Communities. Brussels 2000:1-5.

[3] Drummond MF, Wilson DA, Kanavos P, Ubel P, Rovira J. Assessing the economic challenges posed by orphan drugs. International journal of technology assessment in health care. 2007; 23(1):36-42.

[4] McCabe C, Claxton K, Tsuchiya A. Orphan drugs and the NHS: should we value rarity? BMJ. 2005; 331(7523):1016-9.

[5] McCabe C, Tsuchiya A, Claxton K, Raftery J. Orphan drugs revisited. QJM : monthly journal of the Association of Physicians. 2006; 99(5):341-5.

[6] Desser AS, Gyrd-Hansen D, Olsen JA, Grepperud S, Kristiansen IS. Societal views on orphan drugs: cross sectional survey of Norwegians aged 40 to 67. BMJ. 2010; 341:c4715.

[7] Desser AS, Olsen JA, Grepperud S. Eliciting preferences for prioritizing treatment of rare diseases: the role of opportunity costs and framing effects. PharmacoEconomics. 2013; 31(11):1051-61.

[8] Desser AS. Prioritizing treatment of rare diseases: a survey of preferences of Norwegian doctors. Social science \& medicine. 2013; 94:56-62.

[9] Redelmeier DA, Tversky A. Discrepancy between Medical Decisions for Individual Patients and for Groups. In: Shafir E, ed. Preference, belief, and similarity: Selected writings by Amos Tversky. Cambridge, MA, US: MIT Press 2004:887-93.

[10] McKie J, Richardson J. The Rule of Rescue. Social science \& medicine. 2003; 56:2407-19.

[11] Slovic P, Finucane ML, Peters E, MacGregor DG. The affect heuristic. European Journal of Operational Research. 2007; 177(3):1333-52.

[12] Tversky A, Kahneman D. The Framing of Decisions and the Psychology of Choice. Science. $1981 ; 211(4481): 453-8$.

[13] Tinghög G. The art of saying no : the economics and ethics of healthcare rationing [Doctoral thesis]. Linköping: Linköping University; 2011.

[14] Hamilton DL, Sherman SJ. Perceiving Persons and Groups. Psychological Review. 1996; 103(2):336-55.

[15] Västfjäll D, Slovic P, Mayorga M, Peters E. Compassion fade: Affect and charity are greatest for a single child in need. PloS one. 2014; 9(6). 
[16] Kogut T, Ritov I. The "identified victim" effect: An identified group, or just a single individual? Journal of Behavioral Decision Making. 2005; 18(3):157-67.

[17] Kogut T, Ritov I. The singularity effect of identified victims in separate and joint evaluations. Organizational Behavior and Human Decision Processes. 2005; 97(2):106-16.

[18] Kogut T, Ritov I. The identifiable victim effect: Causes and boundary conditions. In: Oppenheimer DM, Olivola C, eds. The Science of Giving: Experimental Approaches to the Study of Charity. New York: Psychology Press 2011:133-45.

[19] Jenni K, Loewenstein G. Explaining the Identifiable Victim Effect. Journal of Risk and Uncertainty. 1997; 14(3):235-57.

[20] Jonsen AR. Bentham in a box: technology assessment and health care allocation. Law, medicine \& health care : a publication of the American Society of Law \& Medicine. 1986; 14(3-4):172-4.

[21] MacKenzie R, Chapman S, Salkeld G, Holding S. Media influence on Herceptin subsidization in Australia: application of the rule of rescue? Journal of the Royal Society of Medicine. 2008; 101(6):30512.

[22] Lee S, Feeley TH. The identifiable victim effect: a meta-analytic review. Social Influence. 2016; 11(3):199-215.

[23] Baron J. Confusion of Relative and Absolute Risk in Valuation. Journal of Risk and Uncertainty. 1997; 14(3):301-9.

[24] Bartels DM. Proportion dominance: The generality and variability of favoring relative savings over absolute savings. Organizational Behavior and Human Decision Processes. 2006; 100:76-95.

[25] Markowitz EM, Slovic P, Västfjäll D, Hodges SD. Compassion fade and the challenge of environmental conservation. Judgment \& Decision Making. 2013; 8(4):397-406.

Becker GS. The Economic Approach to Human Behavior: University of Chicago Press 1978.

[27] Damschroder LJ, Roberts TR, Zikmund-Fisher BJ, Ubel PA. Why people refuse to make tradeoffs in person tradeoff elicitations: a matter of perspective? Medical Decision Making. 2007; 27(3):266-80.

[28] Dragojlovic N, Rizzardo S, Bansback N, Mitton C, Marra CA, Lynd LD. Challenges in measuring the societal value of orphan drugs: insights from a canadian stated preference survey. The patient. 2015; 8(1):93-101.

[29] Mentzakis E, Stefanowska P, Hurley J. A discrete choice experiment investigating preferences for funding drugs used to treat orphan diseases: an exploratory study. Health economics, policy, and law. 2011; 6(3):405-33. 
[30] Linley WG, Hughes DA. Societal views on NICE, cancer drugs fund and value-based pricing criteria for prioritising medicines: a cross-sectional survey of 4118 adults in Great Britain. Health economics. 2013; 22(8):948-64.

[31] Wiss J, Andersson D, Slovic $P$, Västfjäll D, Tinghög $G$. The influence of identifiability and singularity in moral decision making. Judgment and decision making. 2015; 10(5):492-502.

[32] Rachul C, Toews M, Caulfield T. Controversies with Kalydeco: Newspaper coverage in Canada and the United States of the cystic fibrosis "wonder drug". Journal of Cystic Fibrosis. 2016; 15 (5): 624-629. 


\begin{tabular}{|c|c|c|}
\hline $\begin{array}{c}\text { Version A } \\
\text { Baseline }\end{array}$ & $\begin{array}{c}\text { Version B } \\
\text { Attribute Framing }\end{array}$ & $\begin{array}{c}\text { Version C } \\
\text { Prop. Dominance + Identifiability }\end{array}$ \\
\hline
\end{tabular}

\begin{tabular}{|c|c|c|c|}
\hline $\begin{array}{l}\text { Scenario } 1 \\
\text { (group level, } \\
\text { equal cost) }\end{array}$ & $\begin{array}{ll}- & 100 \text { rare patients } \\
\text { - } & 100 \text { common patients } \\
\text { - } & \text { Indifferent } \\
\end{array}$ & $\begin{array}{ll}- & 10 \text { rare patients } \\
\text { - } & 10 \text { common patients } \\
\text { - } & \text { Indifferent } \\
\end{array}$ & $\begin{array}{ll} & 80 / 80 \text { rare patients } \\
\text { - } & 100 / 10000 \text { common patients } \\
\text { - } & \text { Indifferent } \\
\end{array}$ \\
\hline & $\downarrow$ & $\downarrow$ & $\downarrow$ \\
\hline \multirow[t]{2}{*}{$\begin{array}{l}\text { Scenario } 2 \\
\text { (group level, } \\
\text { unequal cost) }\end{array}$} & $\begin{array}{ll}- & 100 \text { rare patients } \\
\text { - } & 800 \text { common patients } \\
\text { - } & \text { Indifferent } \\
\end{array}$ & $\begin{array}{ll}- & 10 \text { rare patients } \\
\text { - } & 80 \text { common patients } \\
\text { - } & \text { Indifferent } \\
\end{array}$ & $\begin{array}{ll}- & 80 / 80 \text { rare patients } \\
\text { - } & 800 / 10000 \text { common patients } \\
\text { - } & \text { Indifferent } \\
\end{array}$ \\
\hline & $\downarrow$ & $\downarrow$ & $\downarrow$ \\
\hline \multirow[t]{2}{*}{$\begin{array}{l}\text { Scenario } 3 \\
\text { (individual level, } \\
\text { equal cost) }\end{array}$} & $\begin{array}{ll}- & \text { Patient } X \text { (rare) } \\
- & \text { Patient } Y \text { (common) } \\
\text { - } & \text { Indifferent } \\
\end{array}$ & $\begin{array}{ll}- & \text { Deny patient } X \text { (rare) } \\
\text { - } & \text { Deny patient } Y \text { (common) } \\
\text { - } & \text { Indifferent } \\
\end{array}$ & $\begin{array}{ll}- & \text { Eric (rare) } \\
- & \text { Patient Y (common) } \\
\text { - } & \text { Indifferent } \\
\end{array}$ \\
\hline & $\downarrow$ & $\downarrow$ & $\downarrow$ \\
\hline $\begin{array}{l}\text { Scenario } 4 \\
\text { (individual level, } \\
\text { unequal cost) }\end{array}$ & $\begin{array}{ll}- & \text { Patient } X \text { (rare) } \\
\text { - } & \text { Patient } Y+7 \text { (common) } \\
\text { - } & \text { Indifferent }\end{array}$ & $\begin{array}{ll}\text { - } & \text { Deny patient } \mathrm{X} \text { (rare) } \\
\text { - } & \text { Deny patient } \mathrm{Y}+7 \text { (common) } \\
\text { - } & \text { Indifferent }\end{array}$ & $\begin{array}{ll}- & \text { Eric (rare) } \\
\text { - } & \text { Patient } \mathrm{Y}+7 \text { (common) } \\
\text { - } & \text { Indifferent }\end{array}$ \\
\hline
\end{tabular}




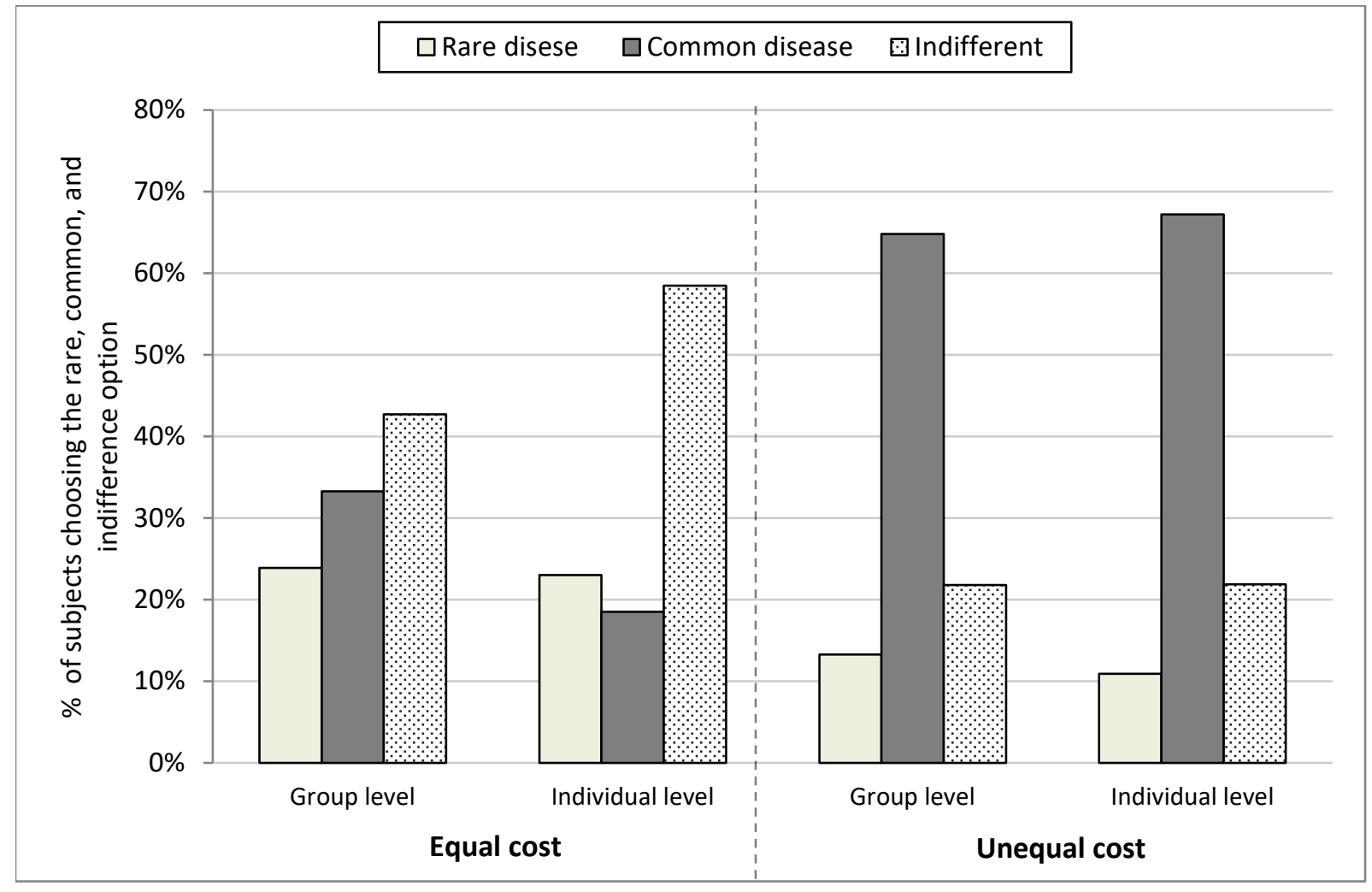




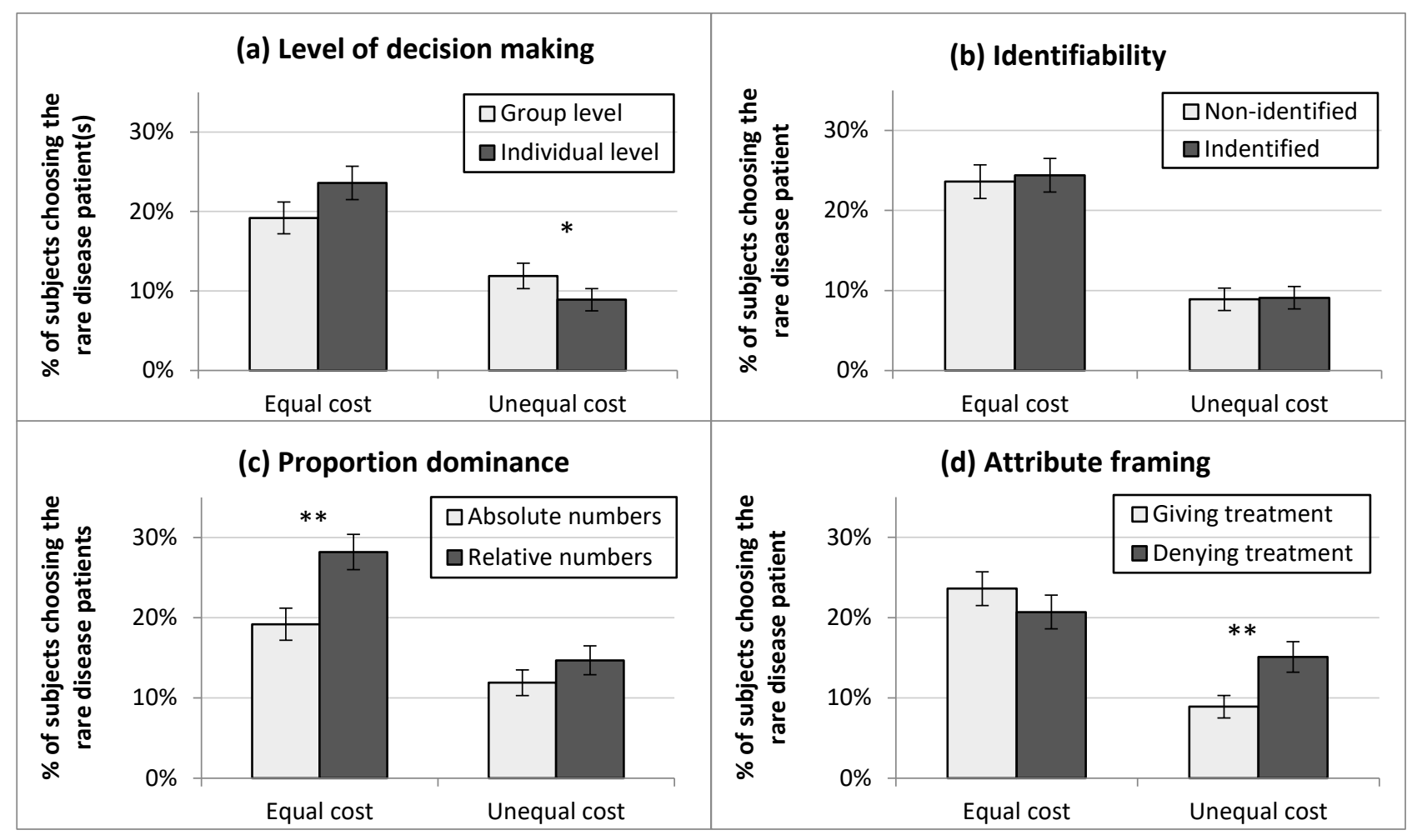

